\title{
LAS CinCO KaTAS DE YAGI MEITOKU
}

Perry Campbell

$\Delta \nabla \mathbf{\Delta}$

UNA FOTOGRAFÍA DE

YAGi Meitoku en 1985, EL AÑO DE SU 75ํㅡㄹ CUMPLEAÑOS.

El MAESTRO Yagi REgaló

ESTA FOTOGRAFÍA A

Paul Babladelis en 1989.

El resto de fotografías son cortesía de Perry Campbell.

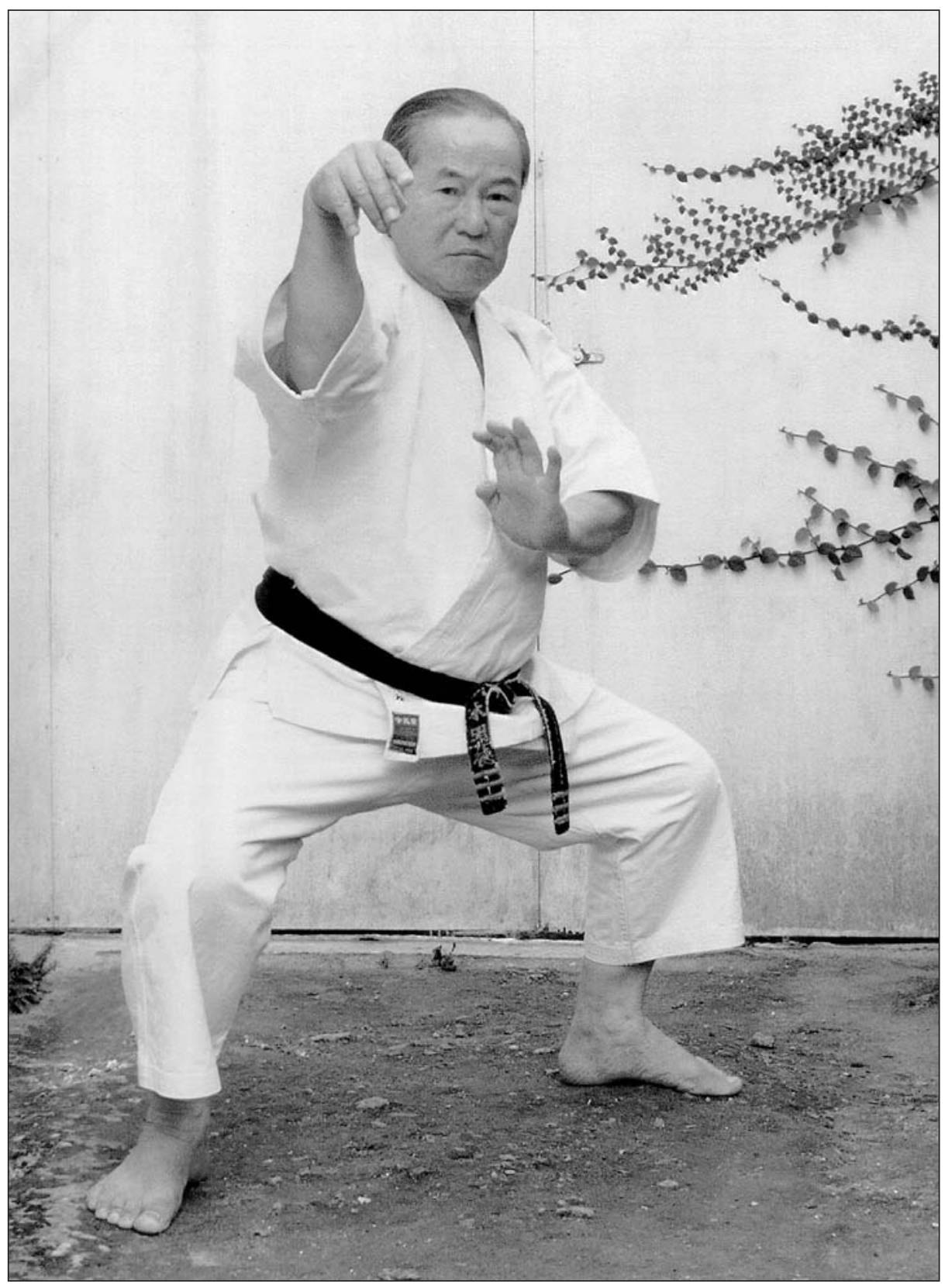




\section{INTRODUCCIÓN}

El fallecimiento del Gran Maestro (Dai Sensei) Yagi Meitoku a los 91 años (7 de febrero de 2003) significó el final de una era en el arte del Goju-Ryu, dejando un gran vacío en la comunidad de artes marciales de Okinawa. El linaje del Sr. Yagi se remonta a las primeras treinta y seis familias chinas que llegaron hace más de seiscientos años al distrito Kume de Naha, en Okinawa, trayendo con ellos, entre muchas otras cosas, sus artes marciales. El amor, dedicación y pasión de Yagi Meitoku por el Kárate Goju-Ryu y su maestro, Miyagi Chojun, radiaba de él. El Sr. Yagi dedicó su vida a enseñar las técnicas avanzadas de Goju-Ryu hasta bien entrados sus 80 años. Dejó cinco katas, las katas Meibuken, como su contribución al Kárate Goju-Ryu: Cielo y Tierra (Tenshi) y los cuatro míticos guardianes que los protegen: Dragón Azul (Seryu), Tigre Blanco (Byako), Gorrión Rojo (Shujaku) y Tortuga Negra (Genbu). Ilusoriamente simples en su forma, y sin embargo fundamentadas en complejas teorías, las katas Meibuken son la evidencia de las prácticas de combate, técnicas, ensayos e innovaciones que Yagi Meitoku desarrolló durante una vida dedicada al estudio.

\section{El SuCESOR DE MiYagi}

Después del fallecimiento de Miyagi Chojun (8 de octubre de 1953), existió un pequeño grupo de individuos que sintió que eran los justos herederos del sistema Goju- Ryu: Higa Seiko, Miyazato Ei'ichi, Miyagi An'ichi, y el hijo de Miyagi Chojun, llamado Kei. A pesar de que Miyagi nunca escogió un continuador ni comunicó su sucesión a ninguno de sus estudiantes antes de su muerte, muy pocos de los líderes del resto de los grandes sistemas de kárate de Okinawa disputaron la demanda que hizo Yagi Meitoku como sucesor. Al final, la responsabilidad de escoger a un continuador cayó sobre los hombros de la mujer e hijos de Miyagi Chojun. De acuerdo con Miyagi Masu (Tsuru), la hija mayor de Miyagi, la elección de Yagi no fue difícil. En una carta traducida en el programa Interviews [Entrevistas] de Ernest Estrada, Tsuru escribía:

Yo y la familia Miyagi le respetamos mucho [a Yagi]. Desde la muerte de mi padre, la familia se ha reunido para debatir quién sucedería a mi padre como cabeza del estilo Goju-Ryu. La decisión se basó en quién había estudiado durante más tiempo, con más dedicación y con mayor lealtad hacia mi padre. Así se decidió que Meitoku Yagi lideraría el estilo Goju- Ryu, y fue entonces cuando decidimos reconocerle oficialmente dándole el traje y el cinturón de kárate de mi padre.

El hijo de Yagi, Meitetsu, confirmó la lealtad de su padre a Miyagi. Meitetsu dijo que podía recordar cada vez que un tifón golpeaba Okinawa mientras Miyagi estuvo vivo:

Mi padre habría ido primero a la casa de Miyagi para cerrar las contraventanas y hacer los preparativos para la tormenta. Sólo después de que la casa de Miyagi estuviera segura habría vuelto para preparar nuestra casa.

Para avalar la decisión de la familia Miyagi de que Yagi Meitoku sería en segundo gran maestro del sistema Goju-Ryu, fue presentado con el traje de Miyagi (dogi). Tsuru dijo: 
Se sentía que el maestro Yagi era la mejor persona para seguir con las enseñanzas de mi padre. Así que se le pidió que continuara sus enseñanzas, y que además las extendiera para que cualquier persona pudiera conocer el estilo Goju-Ryu.

\section{Entrenando CON MiYagi CHOJUN}

Yagi Meitoku tenía 12 años cuando su abuelo le llevó a la escuela de Miyagi Chojun para empezar formalmente su entrenamiento. En aquella época había cinco o seis estudiantes mayores que Yagi, incluyendo Higa Seiko, Nakaima Genkai, Azama Kasai (Nanjo Kiju), Toguchi Seikichi, y Sakiyama Tatsutoku.

El maestro Yagi recordaba que todos hacían muchas repeticiones de ejercicios preparatorios (yobi undo), contando de 100 a 1000 repeticiones de cada técnica. Era un entrenamiento muy exigente y muchos de los nuevos estudiantes se marcharon a otros lugares para buscar entrenamientos más fáciles. En aquellos tempranos días, Miyagi enseñaba únicamente cuatro katas: Sanchin, Seisan, Seiunchin, y Tensho. Éstas eran las katas de mano abierta (kaishu). Dentro de las mismas, Seiunchin y Seisan eran consideradas las katas de entrenamiento, y debían que ser estudiadas concienzudamente para entender el estilo Goju-Ryu. Yagi estuvo entre los primeros estudiantes de Miyagi que aprendieron todas las katas, incluyendo las katas Saifa, Shisochin, Sanseiryu, Seipai, Kururunfa, y Suparinpei, que Miyagi empezó a enseñar cuando Yagi estaba en su primer año de instituto.

El instructor Yagi decía que tardaron de cuatro a cinco años en empezar a aprender las katas. Luego Miyagi les enseñaba una única kata durante dos o más años. Relataba que el entrenamiento de la kata Sanchin era especialmente duro, recordando cómo la gente le reconocía como estudiante de Miyagi sólo por los cardenales que tenía en sus hombros debido a las duras pruebas de Miyagi con dicha kata.

\section{EN sus Últimos AÑos}

Conocí por primera vez al maestro Yagi en 1988. Recuerdo que me preguntaba como sería él. ¿Sería agresivo o impaciente? Había oído historias sobre su impresionante energía. Había roto con frecuencia postes de golpeo (makiwara) a propósito. Cómo tenía poco que ver aparentemente con otros estilos de GojuRyu... Así que fue una sorpresa ver que nada era como lo había esperado. El Sr. Yagi hablaba suavemente y era amigable y tranquilo, como si fuera mi abuelo. Sin embargo, sus ojos eran sagaces y podían cambiar a voluntad para ser temido, exhibiendo su mirada destructiva (koroshi no mei). Los músculos bajo su envejecida piel eran sorprendentemente fuertes y se movía con naturalidad, fluida y velozmente a pesar de su avanzada edad.

En 1990, cuando me fui a Okinawa, era muy obvio que la pasión de Yagi no había menguado. Por entonces ya se había retirado de la enseñanza formal, pero continuaba dando clases a tres grupos de niños cada semana -un grupo sobre el que sorprendentemente tenía poco control-. También instruía a extranjeros por las mañanas. Las visitas de los extranjeros solían durar por lo general dos o tres semanas. Desde que estuve viviendo allí, sin embargo, trabajó conmigo cada mañana y después otra vez por la tarde, seis y siete días a la semana. Además, me permitió participar en las clases de los niños. Durante este periodo, y a pesar de atravesar momentos de poca salud, no sólo vino al dojo a impartir sus enseñanzas dos o tres veces al día, sino que siguió entrenando diariamente. Fue durante este periodo cuando empecé a ver lo extraordinario que era Yagi Meitoku. 
A los 80 años, Yagi era engañosamente ágil y rápido, y todavía poseía una gran fuerza. Pero todavía había más en él. Tocaba regularmente el piano y el violín. Era un maestro en caligrafía. En 1988 se proclamó Campeón de Japón de ajedrez chino (Chunji). En 1989, defendió su titulo y llegó a ser también campeón mundial. No es sorprendente, por tanto, que su maestría en la estrategia fluyese hacia las teorías, técnicas y aplicaciones de sus artes marciales. Esta maestría también impregna las cinco katas Meibuken.

\section{INFUENCIAS}

Algunos han dicho que Sr. Yagi enseñó las katas "exactamente" como Miyagi Chojun se las enseñó a él; sin embargo, esto no es cierto. Tal y como la familia Miyagi pidió, Yagi Meitoku dedicaría toda su vida a estudiar y expandir las enseñanzas de su profesor. Pero existen varios matices y detalles sutiles de las katas del estilo Goju-Ryu que él desarrolló en sus últimos años. Algunas de estas modificaciones vinieron incluso después de que algunos de sus discípulos mayores hubiesen abierto sus propias escuelas. Por lo tanto, no todos sus discípulos directos practican algunas de sus innovaciones.

Además de su maestro, Miyagi Chojun, Yagi reconocía la influencia de dos hombres sobre sus innovaciones, aunque también podría haber habido otros. El primero era su amigo, Ko Shinko, un maestro de las artes marciales chinas nacido en Taiwán, conocido por haber introducido los ejercicios de condición física (kotikitai) en el kárate de Okinawa. El segundo era otro amigo cercano, Taira Shinken, un maestro en armas de combate y presidente de la Ryukyu Kobudo Hozoin Shinkokai. Aunque cada innovación que incorporaba Yagi era sutil, cada una de ellas incrementaba notablemente la fuerza y efectividad de la técnica. Las katas Meibuken no sólo comprenden las técnicas del estilo Goju-Ryu, sino que también incluyen las influencias de estos dos hombres.

La influencia china sobre las katas Meibuken es inmediatamente visible desde el primer movimiento. Yagi Meitoku incorporó el estilo chino de saludo y apertura, donde los pies están separados la anchura de los hombros, las manos forman un triángulo en frente del cuerpo a la altura de la barbilla, con la mano derecha en forma de puño y la izquierda abierta (una representación del carácter chino de la dinastía Ming). Sin embargo, la aportación más significativa del sistema chino es la idea de unir dos katas aisladas para crear un combate entre dos hombres, logrando prácticamente no alterar las katas originales. A un nivel básico, estos enfrentamientos contienen golpeos, patadas, y técnicas de agarre, todos ellos ejecutados en un movimiento continuo desde el principio hasta el final. A niveles más profundos, las aplicaciones son brutalmente eficaces.

\section{BRILLO ENGAÑOSO}

Pienso que son dos aspectos más notables de las katas del Sr. Yagi. Uno es el modo secreto en que se emparejan-Seiryu/Byakko, Shujakku/Genbu, y Tenshi- encajando juntas para ser practicadas como combate entre dos personas. El segundo es que desarrolló estas katas en su cabeza, teniendo en cuenta que la segunda kata de cada conjunto debía ser realizada de manera inversa -como el reflejo de un espejo- para que pudiesen realizarse juntas. Hizo esto sin la ventaja de un compañero que asegurase la sincronización de los movimientos de las katas. Estos son unos claros indicadores de la avanzada comprensión estratégica de Yagi.

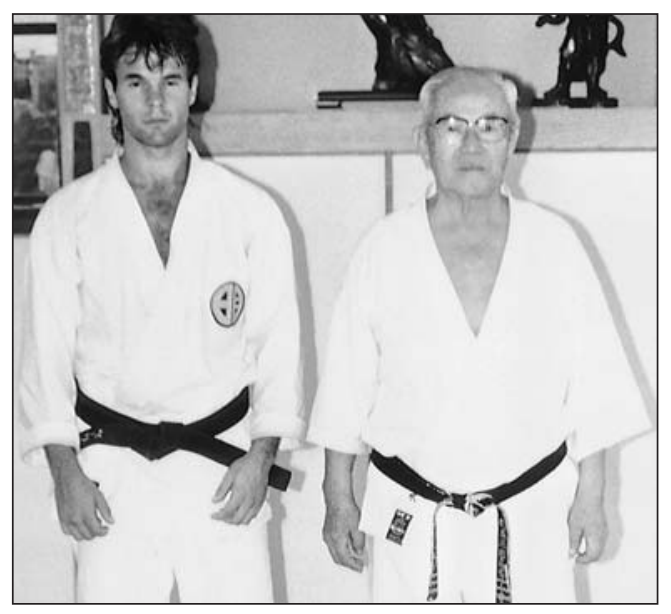




\section{Las Cinco Katas Meibuken}

\section{CIELO Y TIERRA}

Originalmente llamada Fukyo kata Ichi/Ni, Cielo y Tierra (Tenshi) fue la primera kata Meibuken de Yagi. En el libro Okinawan karate-do Goju-Ryu Meibukan escrito por Yagi Meitetsu, Carl Wheeler, y Brock Vickerson (1998), Yagi señalaba, "tuve por primera vez la idea para esta kata tras una visita a Taiwán". Tenshi es una kata que se divide en dos mitades -Cielo y Tierra- que se ensamblan para formar un pequeño combate entre dos personas. La kata se caracteriza por golpes con la mano abierta y con los dedos, golpes dobles, controles de muñeca y barridos. Desarrollada en 1974, Tenshi fue un experimento que demostró el éxito y abrió el camino para las siguientes cuatro katas Meibuken.

\section{DRAGÓN AZUL}

El Dragón Azul (Seiryu) es el guardián mítico del oeste. La kata se caracteriza por utilizar golpes con la rodilla, golpes percutantes, y potentes rotaciones de cintura y cadera para generar energía tanto para bloqueos como para golpeos. Dragón Azul se empareja con Tigre Blanco para formar el combate entre dos personas. Por tanto, contiene los ataques y contraataques para la kata Tigre Blanco.

La rotación de las caderas y de la cintura en la combinación de parada con muñeca, golpe y parada baja se realiza alrededor del eje central del cuerpo, con el cuerpo erguido. El Sr. Yagi usaba con frecuencia un tridente pequeño (sai) como modelo para demostrar la rotación. Agarrándolo perpendicularmente al suelo, con el mango hacia arriba, rotaba el sai en el sentido de las agujas del reloj y al contrario en su mano... Las hojas del sai, según rotaban, mostraban claramente el movimiento que él quería.

\section{Tigre BLANCO}

El Tigre Blanco (Byakko) es el mítico guardián del este. Las técnicas de esta kata son las contrarias a las de Dragón Azul. Tigre Blanco se caracteriza por potentes técnicas de golpeo utilizando movimientos hacia arriba y hacia abajo para crear energía. Esto se logra descendiendo rápidamente desde una posición alta a una posición baja, y después volviendo a la posición alta. La energía generada por medio de esta elevación y descenso se muestra efectiva contra las formas de Dragón Azul, que como se mencionó utilizan la rotación. La kata Tigre Blanco también introduce defensas contra un ataque a la rodilla.

\section{GORRIÓN ROJO}

El Gorrión Rojo (Shujakku) es el mítico guardián del norte de Okinawa. La kata se caracteriza por utilizar técnicas de golpeo con la mano abierta, fintas, combinaciones rápidas de mano y pie, escapes de agarres y ataques mediante bloqueo. Cuando se trabaja como combate entre dos personas con su kata compañera, Tortuga Negra, los movimientos de Gorrión Rojo son ligeros y veloces -como el pájaro del que recibió su nombre la kata- y eluden los agarres de Tortuga Negra.

Curiosamente, el inicio del movimiento final de Gorrión Rojo es el mismo que el paso al penúltimo movimiento de la versión original de la kata Tensho de Miyagi -Heishu Kata Tensho- que recuperó Yagi a principios de 1991. 


\section{LA TORTUGA Negra}

La Tortuga Negra (Genbu) es el mítico guardián del sur. Esta kata se terminó en 1990 y es la última kata del Sr. Yagi. Siendo la compañera de Gorrión Rojo, Tortuga Negra se caracteriza por el uso de posiciones bajas y sólidas para bloquear y golpear. Pero a veces el practicante se eleva a una posición más alta para ejecutar un golpe devastador. La kata también incorpora agarres. Cuando se practica con Gorrión Rojo, los agarres y las contras fluyen con soltura entre los golpes. Las técnicas de esta kata son fuertes y poderosas.

El último movimiento de Tortuga Negra también es el movimiento final del Tensho de Miyagi -Heishu Kata Tensho-. De acuerdo con Yagi, los dos últimos movimientos son realmente un ejercicio chino para incrementar la longevidad. Sus aplicaciones marciales, sin embargo, son la técnica de lucha "Full Nelson" y su contra-ataque.

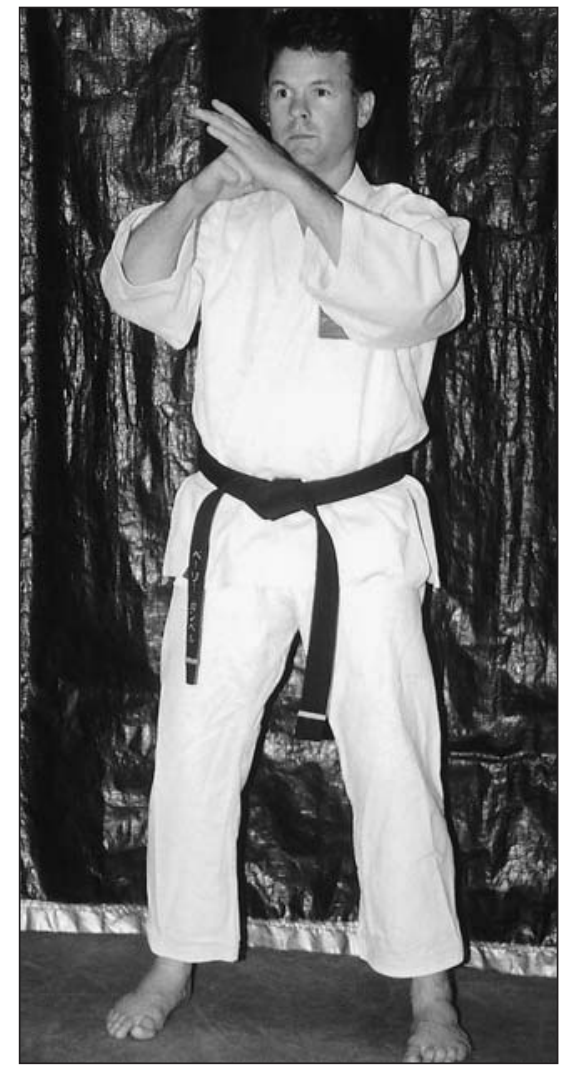

Tanto Tortuga Negra

como Gorrión Rojo comienzan con un saludo formal.

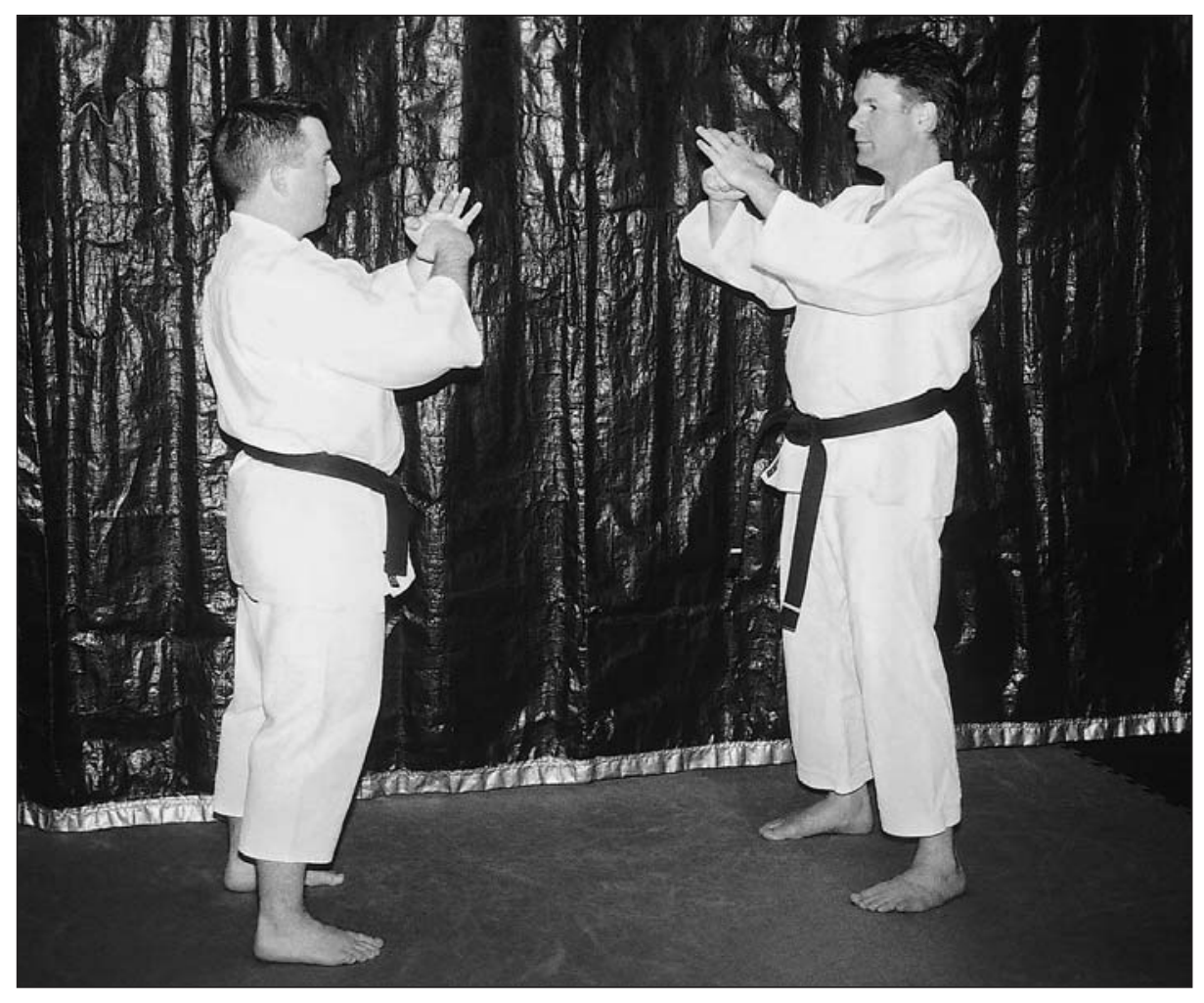

\section{Tortuga Negra y}

\section{Gorrión Rojo}

(Genbu y Shujakku)

Los primeros movimientos de estas dos katas con sus correspondientes secuencias con el compañero.
Los compañeros se complementan en las katas Tortuga Negra y Gorrión Rojo. 
Tortuga Negra

Tortuga Negra y

\section{Gorrión Rojo}

Continuación de la página anterior
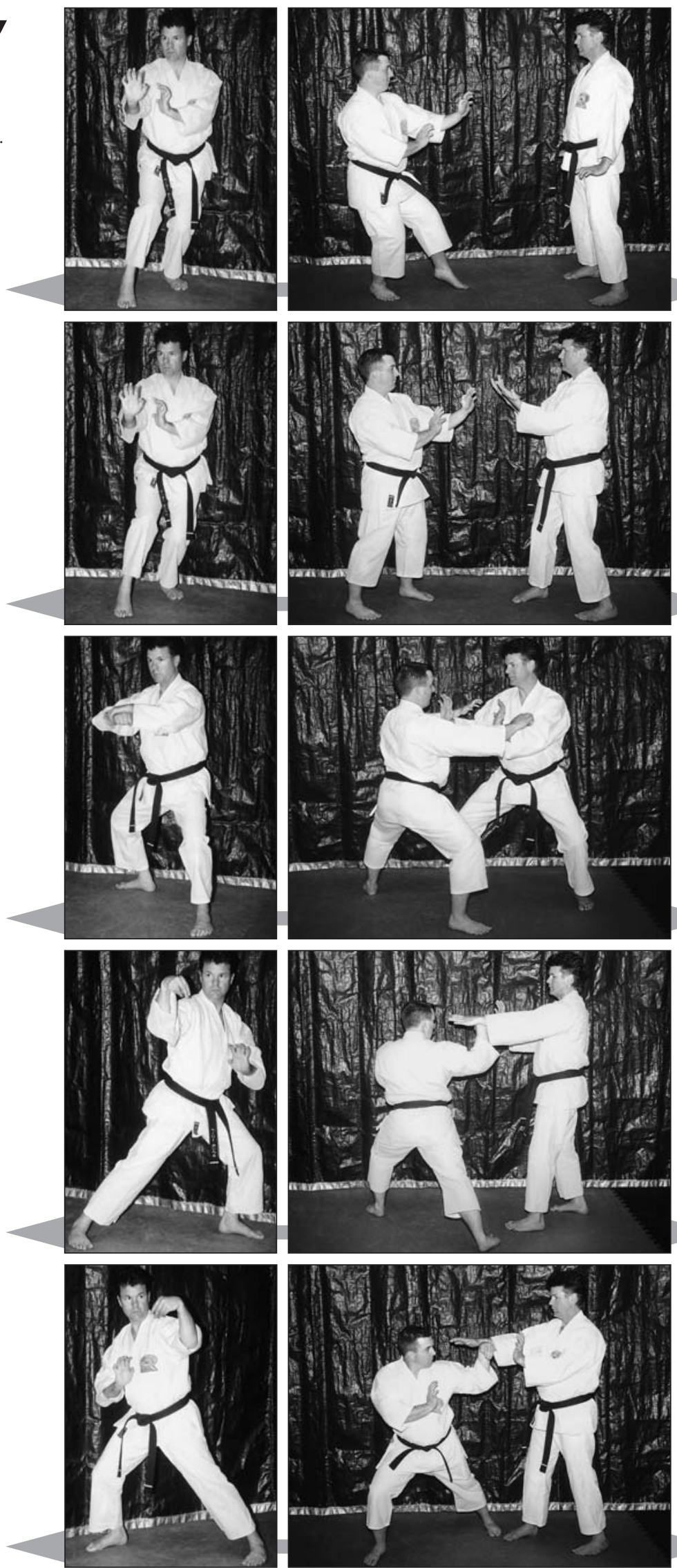

Gorrión Rojo
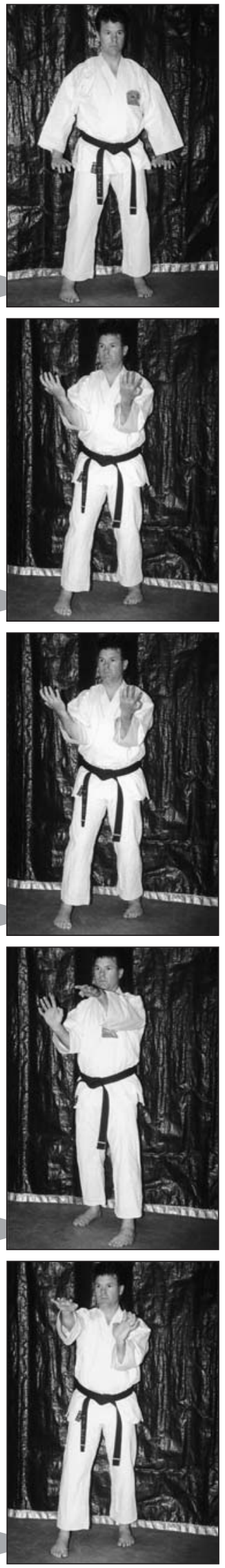
Tortuga Negra
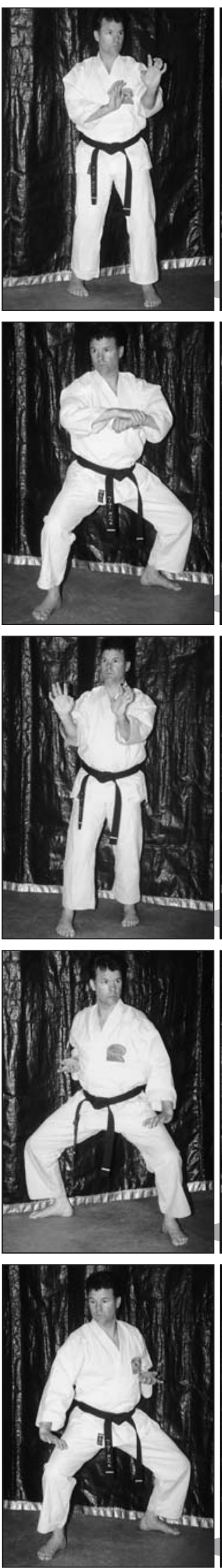

Tortuga Negra y Gorrión Rojo
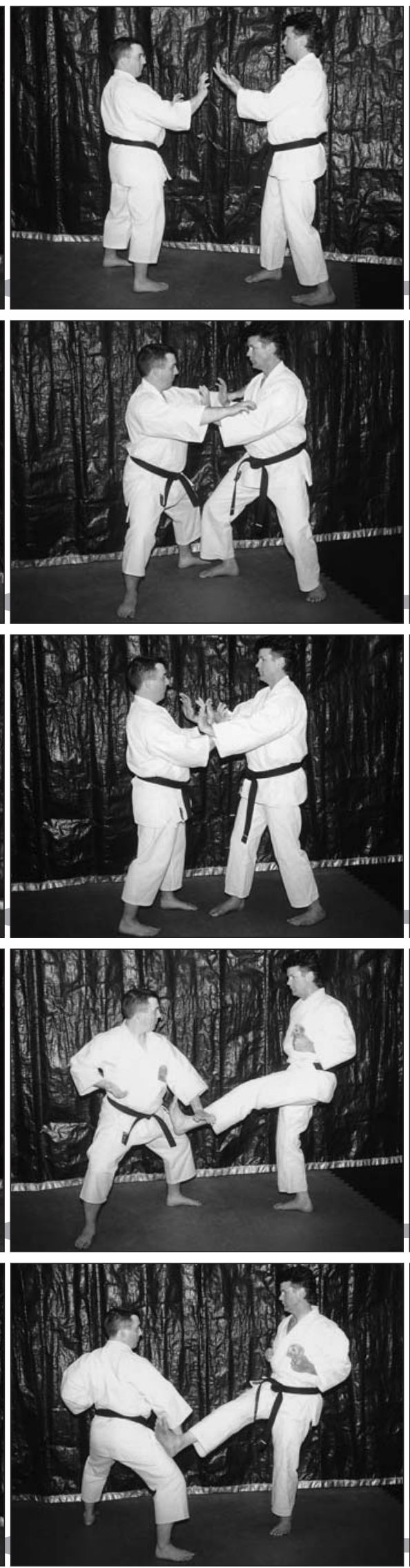

Gorrión Rojo
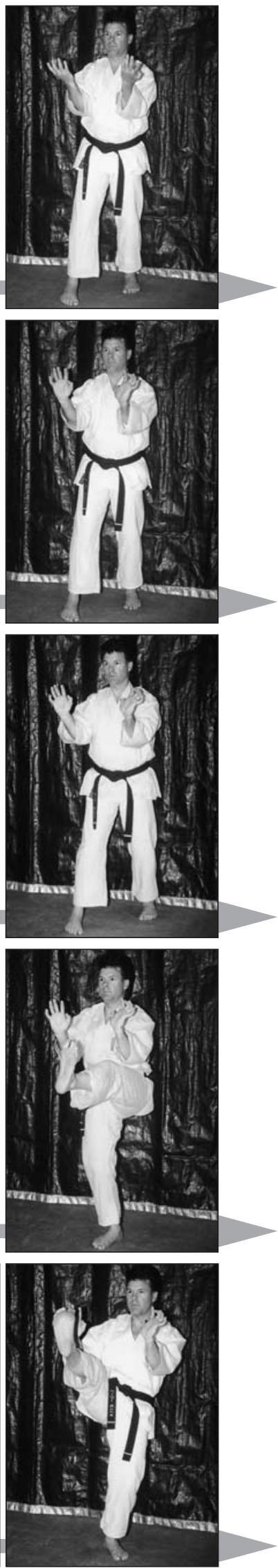
Dragón Azul y Tigre Blanco

\section{Dragón Azul y}

Tigre Blanco

(Seiryu y Byakko)

Los primeros seis movimientos

de estas dos katas con sus

correspondientes secuencias con el compañero, comenzando con el saludo formal.

Tigre Blanco
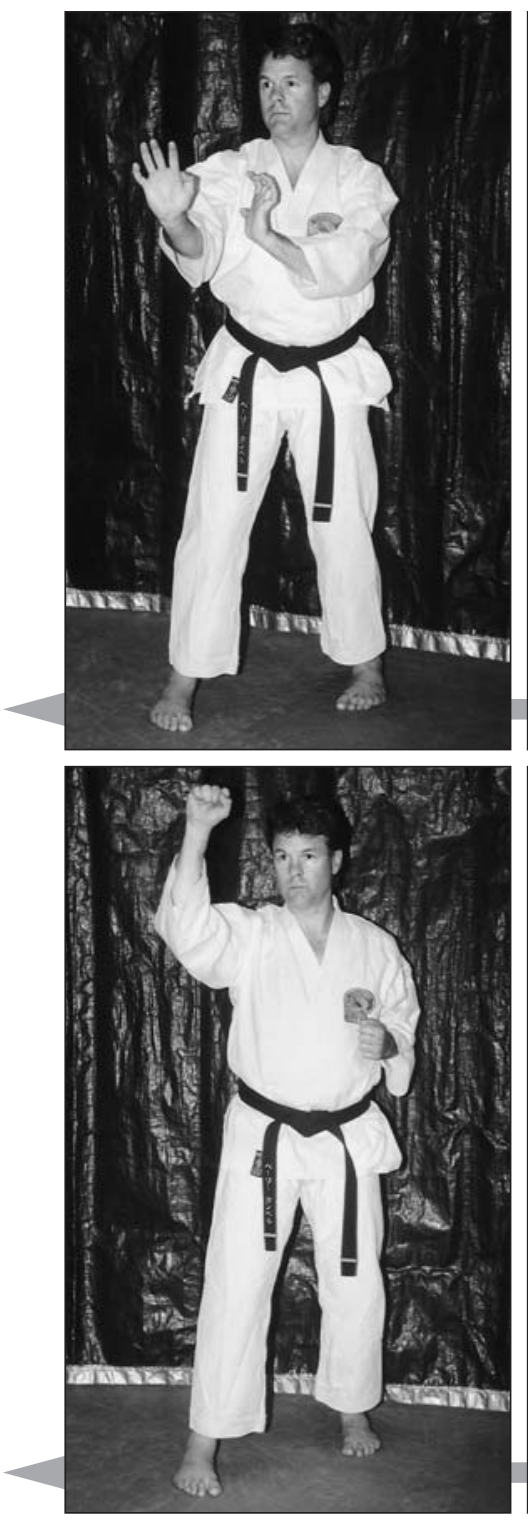
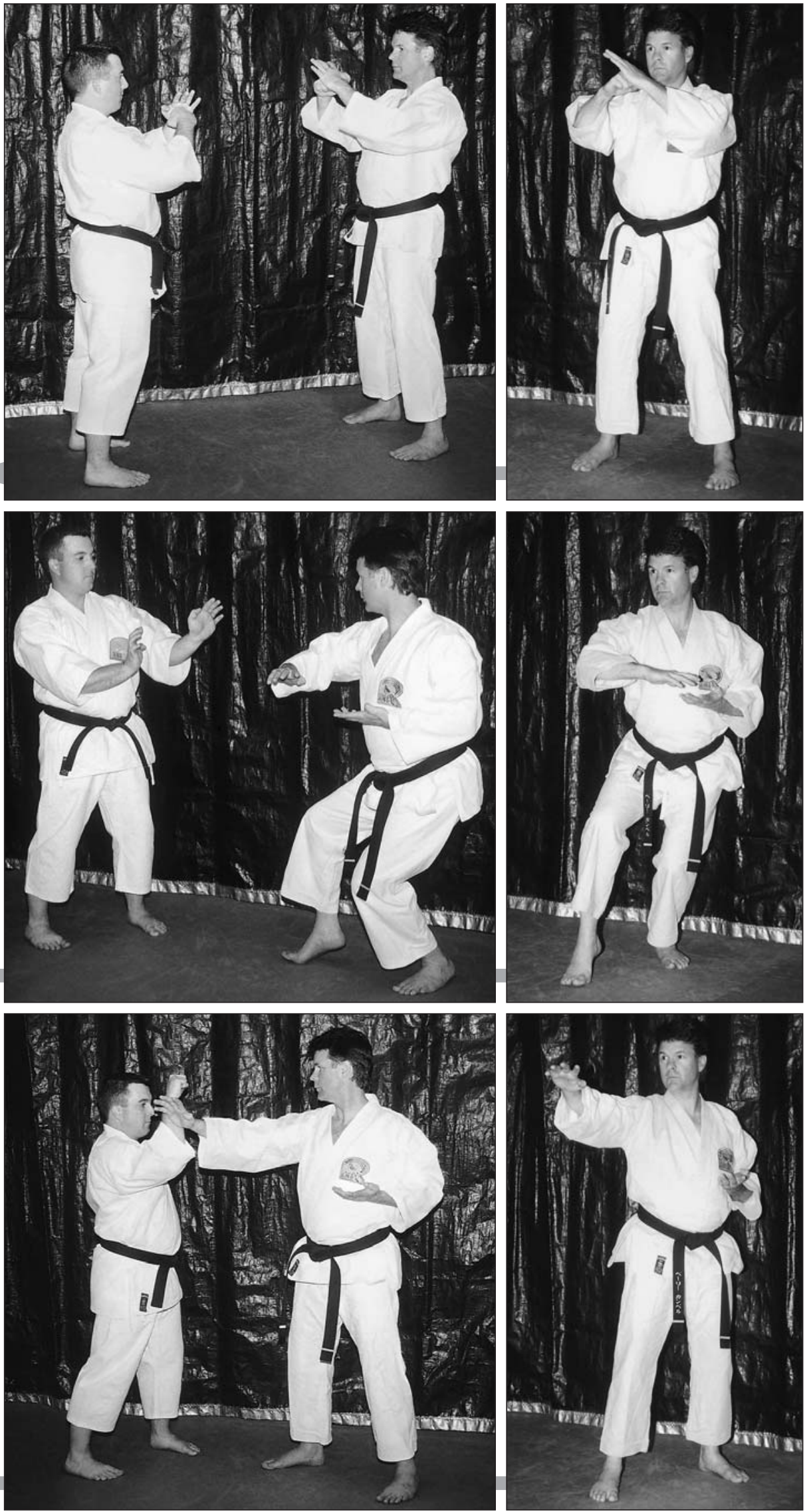

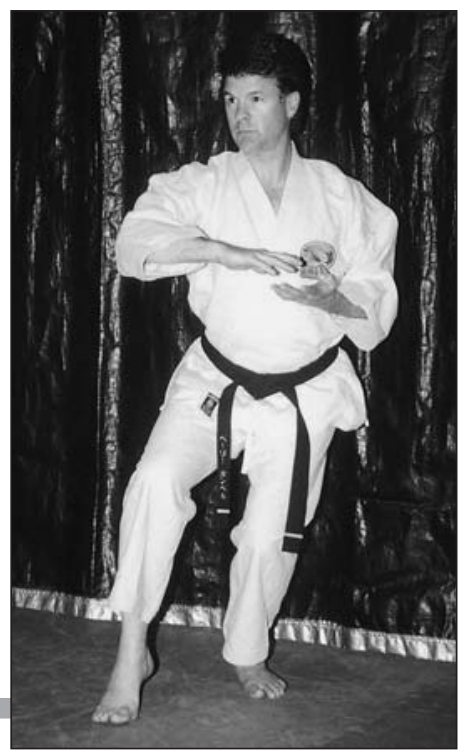

Dragón Azu

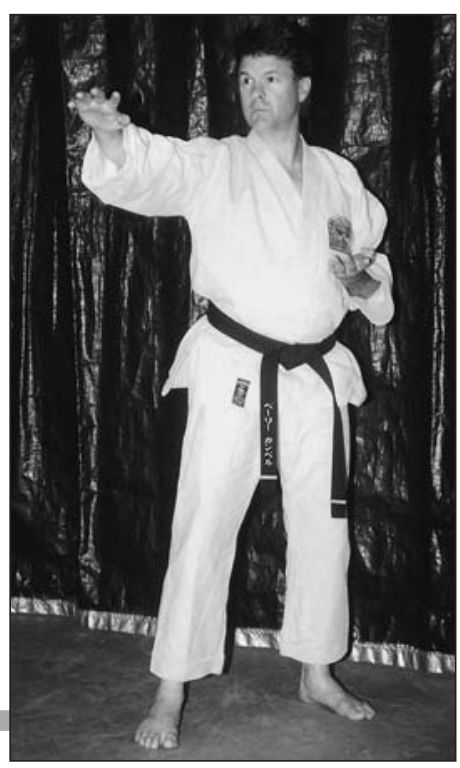


Tigre Blanco
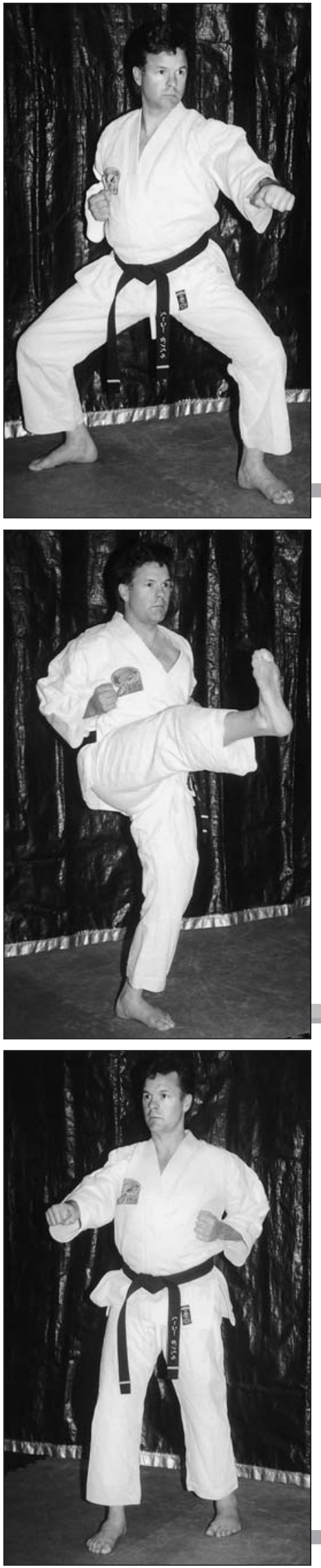

Dragón Azul y Tigre Blanco
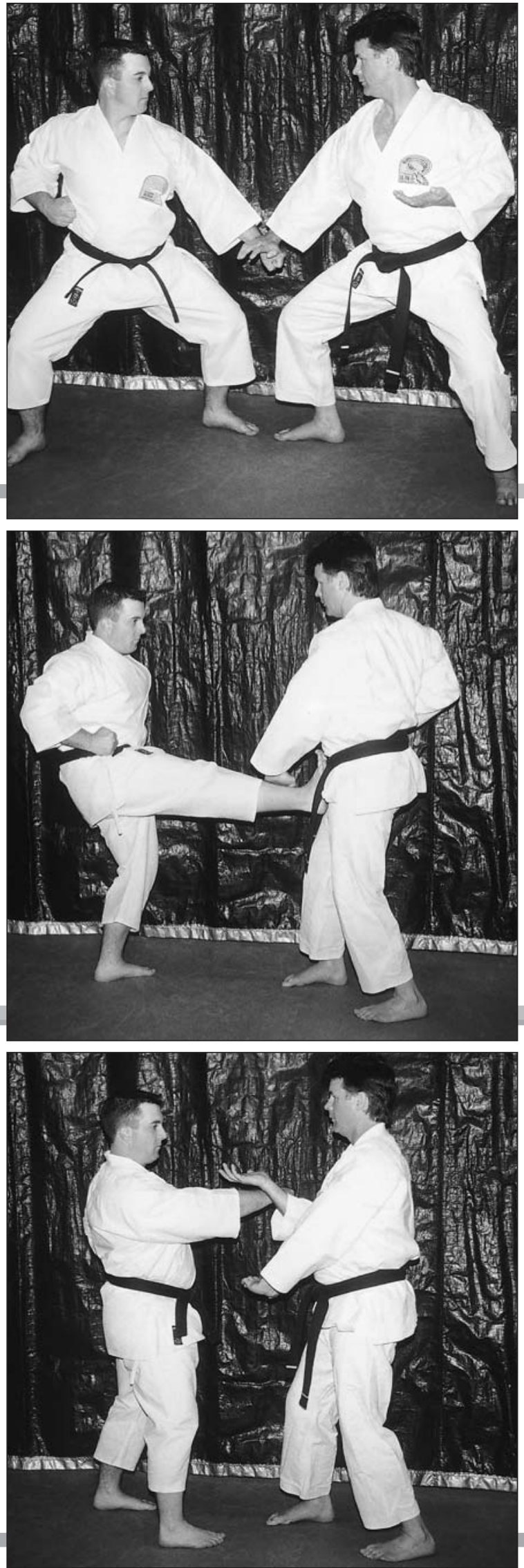

Dragón Azul
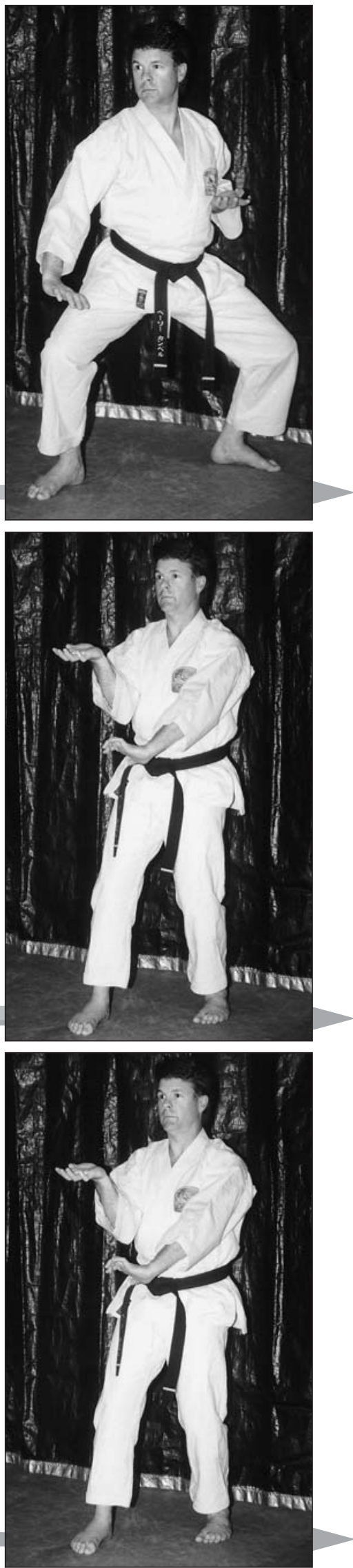

Revista de Artes Marciales Asiáticas 9 Volumen 1 Número 1 (62-73) - 2006 


\section{Aplicaciones}

\section{avanzadas de}

la kata Cielo

\section{y Tierra}

(Tenshi)

Primer movimiento de la kata Tehshi y su aplicación.

Los dos últimos movimientos de la kata Tehshi y su posible aplicación.
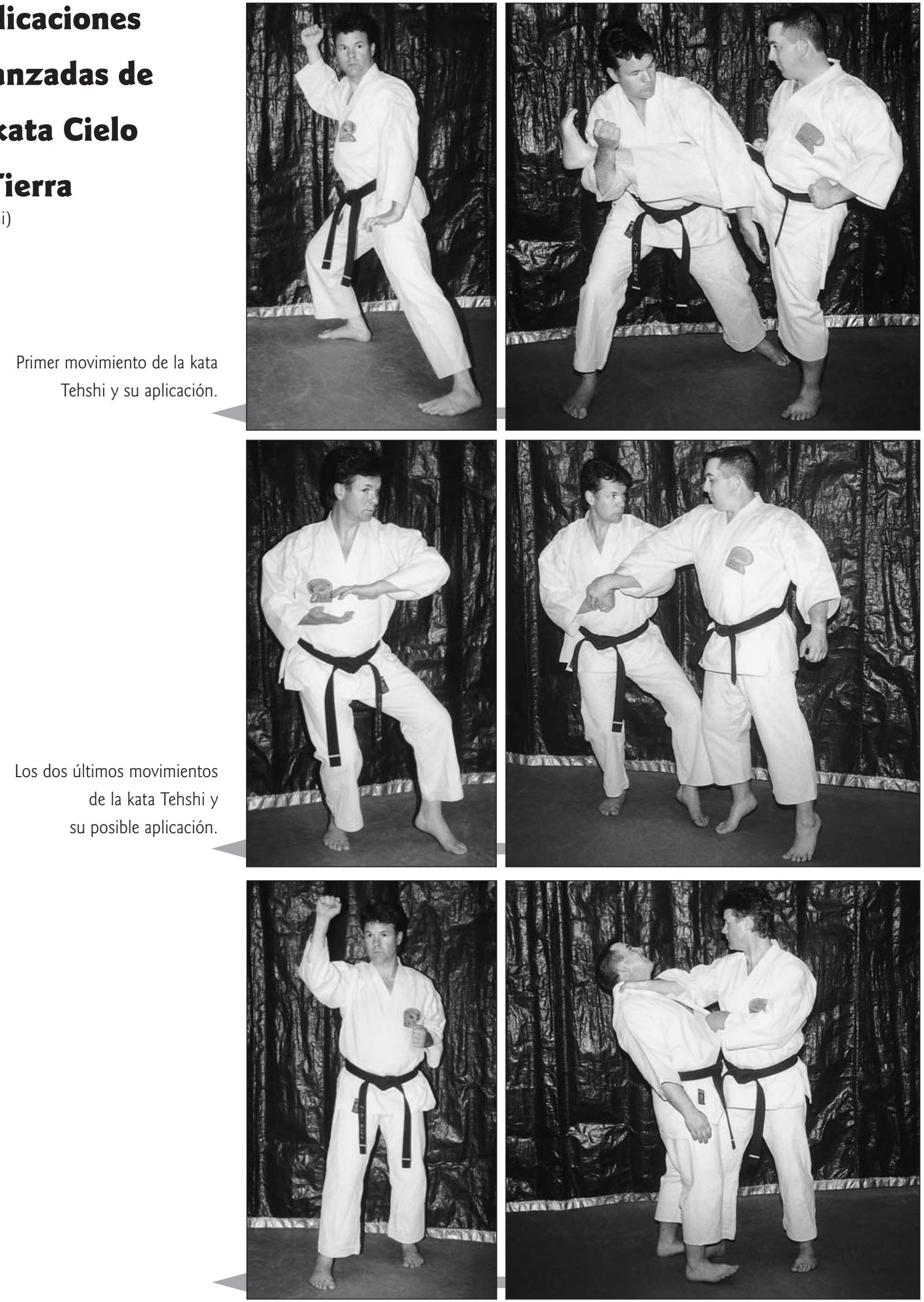

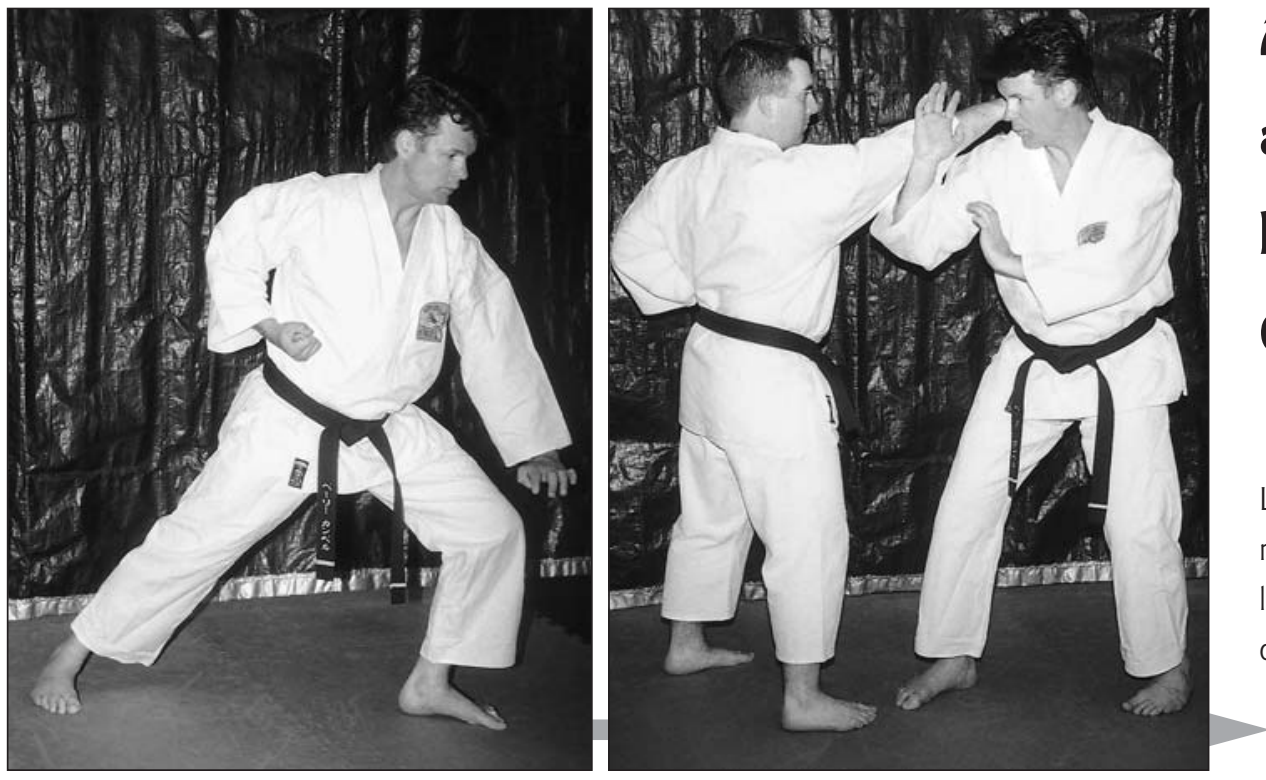

\section{Aplicaciones}

\section{avanzadas de}

\section{la kata}

\section{Gorrión Rojo}
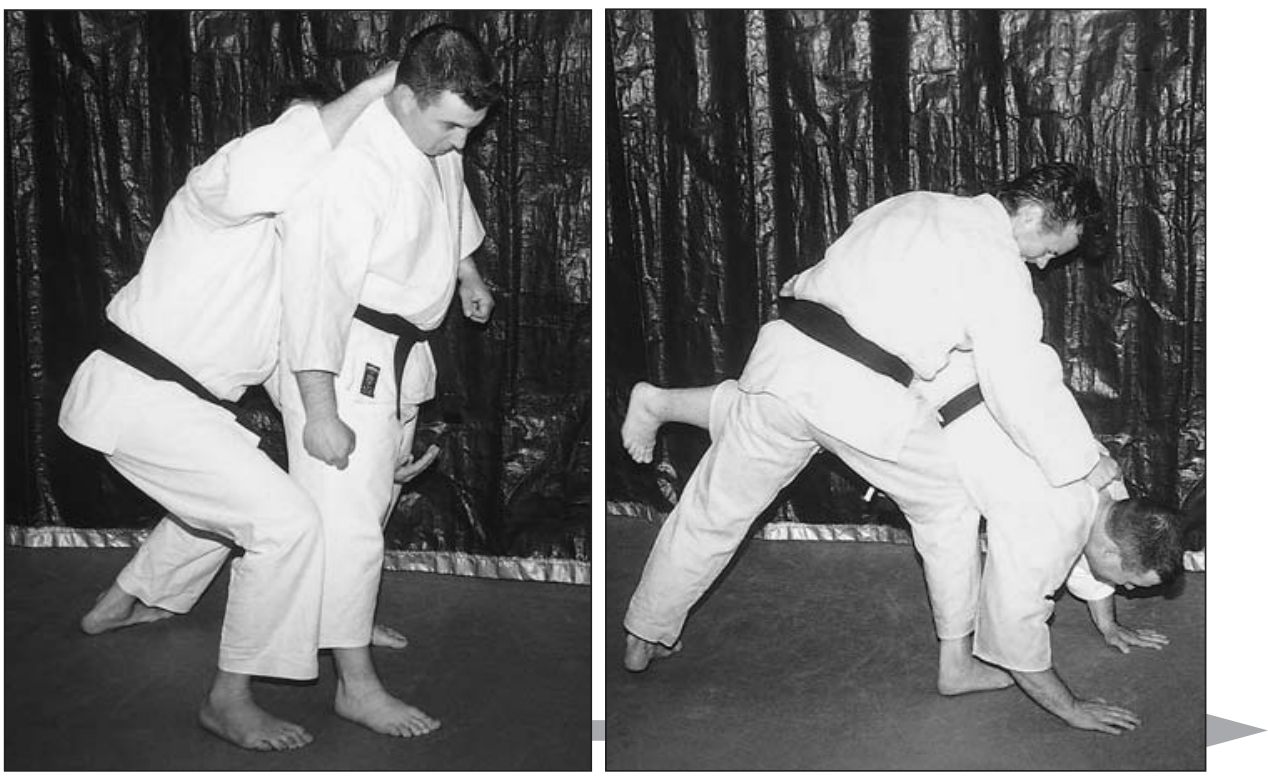

La fotografía de la izquierda muestra el movimiento de la kata en solitario, seguida de una serie que muestra un derribo.

Finta y finalización con derribo.

\section{EL LEGADO}

Yagi Meitoku y sus contemporáneos forman parte de una época que ha sido denominada "la Edad de Oro del kárate". Con sus fallecimientos, los últimos restos de esta época están desapareciendo también. Sin embargo, no todo se ha perdido. Junto con los discípulos directos de Yagi Meitoku, continuadores de sus enseñanzas, el sensei Yagi ha dejado en las katas Meibuken un significativo legado compuesto por algunos de sus conocimientos, técnicas, teorías, e innovaciones.

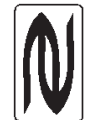

